\title{
Reducing health risks of physical inactivity by cardiac rehabilitation
}

Rainer Spiegel

In her Research Highlight (King, A. Public health: Health risks of physical inactivity similar to smoking. Nat. Rev. Cardiol. 9, 492 [2012]), ${ }^{1}$ Alexandra King refers to the impact of health risks related to physical inactivity. Lack of physical exercise accounts for approximately 1 in 10 deaths annually, and up to $10 \%$ of noncontagious diseases such as type 2 diabetes mellitus, coronary heart disease, and breast or colon cancer. ${ }^{2}$ Physical inactivity is a risk factor for many diseases. However, even a formerly inactive patient who has been diagnosed with a disease such as chronic heart failure (CHF) can substantially benefit from becoming physically active, which is of particular relevance for cardiac rehabilitation. As a physician in a rehabilitation hospital, I regularly see patients who used to be physically inactive making their first steps towards lifestyle changes such as engaging in physical exercise during hospitalization.

Both the Research Highlight by King ${ }^{1}$ and studies published in the medical literature have important practical implications for cardiac rehabilitation. For example, a prospective cohort study involving healthy participants in Taiwan demonstrated that even a small amount of regular exercise (92 min per week) led to a significant increase in life expectancy. ${ }^{3}$ In addition, evidence exists that supervised exercise results in better outcomes than primary stenting in patients with peripheral artery disease. ${ }^{4}$ This finding supports an association between exercise and improved vascular endothelial function owing to reduced intima-media thickness. ${ }^{5}$ Anabolic effects and improved muscular function have been demonstrated with exercise in patients with advanced CHF. ${ }^{6}$ Additional evidence stems from an exerciserelated reduction of E3 ubiquitin-protein ligase TRIM63 (also known as MuRF-1) in the setting of $\mathrm{CHF}^{7}$ MuRF-1 contributes to proteolysis in muscle wasting, to which patients with $\mathrm{CHF}$ are particularly vulnerable. Evidence also exists that exercises incorporating balance and strength training lead to a significant reduction in the number of falls among healthy elderly people (aged $\geq 70$ years). ${ }^{8}$ Balance and strength training will also prevent muscular atrophy, osteoporosis fractures, and secondary illness owing to inactivity after a fall.

In my daily clinical routine, I frequently make the discovery that formerly physically inactive patients with insulin-dependent type 2 diabetes require fewer insulin units after they start exercising. Similarly, patients participating in individualized exercise programs tend to show improvements in the 6-min walk test, pulse oxymetry, and arterial blood gases. Therefore, supporting patients in the continuation of physical exercise after their release from the rehabilitation hospital is a vital component of care.

Klinik Adelheid Rehabilitation Hospital, Internal Medicine Ward, Höhenweg 71, 6314 Unterägeri, Switzerland. rainer.spiege/@klinik-adelheid.ch

\section{Competing interests}

The author declares no competing interests.

1. King, A. Public health: Health risks of physical inactivity similar to smoking. Nat. Rev. Cardiol. 9, 492 (2012)

2. Lee, I. M. et al. Effect of physical inactivity on major non-communicable diseases worldwide: an analysis of burden of disease and life expectancy. Lancet 380, 219-229 (2012).

3. Wen, C. P. et al. Minimum amount of physical activity for reduced mortality and extended life expectancy: a prospective cohort study. Lancet 378, 1244-1253 (2011).

4. Murphy, T. P. et al. Supervised exercise versus primary stenting for claudication resulting from aortoiliac peripheral artery disease: six-month outcomes from the claudication: exercise versus endoluminal revascularization (CLEVER) study. Circulation 125, 130-139 (2012).

5. Pahkala, K. et al. Association of physical activity with vascular endothelial function and intimamedia thickness. Circulation 124, 1956-1963 (2011).

6. Höllriegel, R. et al. Anabolic effects of exercise training in patients with advanced chronic heart failure (NYHA IIIb): impact on ubiquitin-protein ligases expression and skeletal muscle size. Int. J. Cardiol. http://dx.doi.org/10.1016/ j.ijcard.2012.03.083.

7. Gielen, S. et al. Exercise training attenuates MuRF-1 expression in the skeletal muscle of patients with chronic heart failure independent of age: the randomized Leipzig Exercise Intervention in Chronic Heart Failure and Aging catabolism study. Circulation 125, 2716-2727 (2012).

8. Clemson, L. et al. Integration of balance and strength training into daily life activity to reduce rate of falls in older people (the LiFE study): randomised parallel trial. BMJ 345, e4547 (2012). 